\title{
A129 EXPRESSION OF CD62L, CD27 AND CD11B DEFINE A MARKED CYTOTOXIC EFFECTOR ROLE FOR NK CELLS IN RHEUMATOID ARTHRITIS
}

Mónica Abreu, ${ }^{1,2}$ Helena Carvalheiro, ${ }^{1}$ Cátia Duarte, ${ }^{2,3}$ José António Pereirada-Silva, ${ }^{2,3}$ Margarida Souto-Carneiro, ${ }^{1,2}$ Paulo Rodrigues-Santos ${ }^{1,2}{ }^{1}$ Center for Neuroscience and Cell Biology, University of Coimbra, Coimbra, Portugal; ${ }^{2}$ Faculty of Medicine, University of Coimbra, Coimbra, Portugal; ${ }^{3}$ Rheumatology Service, Coimbra University Hospitals, Coimbra, Portugal

\subsection{6/ard.2010.148999.5}

Natural killer (NK) cells play a crucial role in the innate immune response. In the absence of prior activation they are capable of rapidly produce interferon $\gamma(\operatorname{IFN} \gamma)$ and other immunoregulatory cytokines, as well as lyse specific target cells. Previous studies suggested a dual role for NK cells in rheumatoid arthritis (RA) and limited their characterisation to the surface expression of CD56/CD16. However, the newest information on the heterogeneity and complexity of this cell population has deemed such classification insufficient. In an effort to shed a new light into the possible roles of NK cells in RA in the present study the authors have dissected the heterogeneity of NK by looking into the expression of CD27, CD11b and CD62L as well as the production of pro-inflammatory cytokines in the peripheral blood of RA patients.

Peripheral blood NK cells from RA patients $(n=10)$ and healthy donors $(n=10)$ were analysed by flow cytometry, for cell surface expression of CD56, CD16, CD27, CD11b and CD62L, intracellular cytokine production of IFN $\gamma$ and tumour necrosis factor $\alpha$ (TNF $\alpha)$ and degranulation (CD107a).

This data indicate that a higher percentage of CD56 bright CD27 CD11b and CD56 dim CD27 CD11b NK cells are present in RA when compared to healthy donors indicating lower maturation status of NK cell compartment, probably due to chronic enhanced stimulation and proliferation. Regarding NK cell CD62L expression, general reduction was found in RA patients, particularly in CD56 $6^{\text {bright }} \mathrm{NK}$ subset. Better ability for production of IFN $\gamma$ in CD56 ${ }^{\text {bright }} \mathrm{CD} 16$ and CD56 ${ }^{\text {dim }} \mathrm{CD} 16 \mathrm{NK}$ cells was observed in RA patients $(28.4 \% \pm 7.1 \%$ vs $11.3 \% \pm 7.9 \%$ 
and $52.6 \% \pm 11.5 \%$ vs $28.0 \% \pm 17.3 \%$, respectively). Similar results were obtained for TNF $\alpha(26.2 \% \pm 6.1 \%$ vs $14.0 \% \pm 6.7 \%$ and $55.6 \% \pm 8.9 \%$ vs $29.5 \% \pm 14.5 \%$, respectively). Increased degranulation, based on upregulation of CD107a expression, observed in RA patients CD56 ${ }^{\text {dim }}$ CD16 effector NK cells (41.8\% vs $25.9 \%$ ) could be connected to chronic stimulation in disease.

Taken together, this results suggest an important role of CD56 ${ }^{\text {bright/dim }}$ CD27 CD11b NK-cell subsets in RA. These NK cells are potent producers of pro-inflammatory cytokines and thus capable of massive destruction in a chronic inflammation scenario. In line with previous studies, lower expression of CD62L in different RA NK subsets correlates with increased cytotoxicity making NK cell privileged targets for immune therapy. 